\title{
Research on Departmental Collaborations in the Local Government Energy Conservation
}

\author{
YU Wensong ${ }^{1}$, WANG Shujun ${ }^{1}$, CAI Xiaoshen ${ }^{2, a}$ \\ ${ }^{1}$ School of Marxism, Dalian University of Technology, No.2 Linggong Road, Dalian, China \\ ${ }^{2}$ Faulty of Humanities and Social Sciences, Dalian University of Technology, No.2 Linggong Road, Dalian, China
}

\begin{abstract}
The scientific and reasonable departmental collaboration is the key to effective energy conservation work carried out by local governments. This study collects energy-saving policy texts and energy efficiency data of 121 local governments, uses data mining, social network analysis, and related analysis methods to analyze the departmental collaborations in local government energy-saving, and reveals the main problems in departmental collaborations. The results show that: from the structural law, the collaborations with the Department of Water Resources should be strengthened, and the collaborations with Department of Culture and Tourism and Department of Education should be reduced, which can improve energy efficiency. The main problem of current departmental collaborations is that Department of Water Resources is facing obvious lack of collaborations, and the collaborations between relevant departments and Department of Water Resources should be strengthened.
\end{abstract}

\section{Introduction}

In 2019, China consumed 4.86 billion tons of standard coal and emitted 9.429 billion tons of carbon dioxide. It has been the country with the largest energy consumption and carbon dioxide emissions in the world for 14 consecutive years. It not only exceeds the total amount of the United States and the European Union, but also accounts for the world's energy $75 \%$ of the net increase in consumption, which is a serious threat to sustainable development. Energy conservation involves a wide range of fields. Traditional bureaucratic administration disperses government functions into multiple departments, and improves administrative efficiency by refining the government's professional division of labour. However, the increasing demand for public services has led to the continuous expansion of the scale of the government, and the increased pyramid structure has resulted in low administrative efficiency. In the process of shifting to a flat structure, New Public Management introduced a competition mechanism between government departments to improve management efficiency. At the same time, it focused on cooperation with enterprises and saved government costs through the introduction of market mechanisms. However, departmentalism that prioritizes the interests of departments has emerged, and the fragmentation of administrative management has led to inability to deal with complex public issues and low management efficiency. Zadek pointed out that solving complex

\footnotetext{
a Corresponding author: cxshen99@163.com
}

ecological and environmental problems must rely on collaborative governance [1], using collaboration as a means to break departmental functional boundaries and improve the administrative efficiency of cross-domain public issues [2]. From the perspective of collaboration, this study analyzes the local government departmental collaborations in energy conservation, reveals the main problems of government collaboration, and is of great significance to the construction of local government energy conservation systems.

Policy is an important basis for the government departmental collaboration. Scholars at home and abroad use policy as an important path to study departmental collaboration. Meijers and Stead believe that crossborder public issues exceed the scope of responsibility of a single government department and require departmental collaboration to solve [3]. Matei and Dogari believe that due to conflicts of interest between government departments, departmental collaborations cannot be carried out smoothly, and attention should be paid to balancing the conflicts of interests of departments [4]. Hoel found that good departmental collaborations are beneficial to improve the efficiency of policy implementation [5]. Chinese scholars mainly conduct quantitative research on policy texts: First, statistics on external characteristics. Scholars make statistics on policies jointly formulated by government departments in energy conservation based on external characteristics such as the department, time, and types of laws and regulations. The second is to formulate departmental 
network analysis. Because the statistics of policy external characteristics cannot visually display and analyze the collaboration relationship between policymaking departments, Cao Xiaojuan and Li Jiawei use social network analysis methods to analyze the collaboration of formulating departments [6]. The third is to formulate research on the structural law of departmental collaborations. Because the departmental collaboration is difficult to establish a connection with energy efficiency, Li Jiaxue and Zhang Guoxing first assign values to the policy text according to the level and type of laws and regulations for Policy-making departments, and use the value as the policy strength of the policy text. Secondly, whether there is collaboration between the two formulating departments as a binary variable, and multiplying the policy strength by the binary variable as the degree of collaboration among formulating departments in the policy text. According to all relevant policy texts in a certain year, the formulating department can be obtained. Finally, a regression analysis of the total collaboration degree and energy efficiency data between the formulation departments in different years is carried out to reveal the structural law of the formulating departmental collaboration [7]. Generally speaking, the predecessors' researches on the departmental collaboration of governments are relatively comprehensive.

However, there are still shortcomings in the quantitative researches on the departmental collaboration of governments. First, it pays attention to the research on the collaboration of policy-making departments and lacks the research on the collaboration of policy enforcement departments. Second, it pays attention to the research on departmental collaborations of the central government, but lacks of researches on departmental collaborations of the local government. Existing researches mainly conduct statistical analysis on the collaboration of central government departments in different periods, but lacks statistical analysis on the collaboration of different local government departments in the same period, lacks the application of synergy theory, and cannot find the structural laws that determine the development and change of the system. Synergy theory is a theory that explains the movement laws of a dynamic system from disorder to order. If individuals in the system produce order parameters in the process of self-organization, then the movement process will have a structural law, which will regulate the actions of individuals, namely the slaving principle [8], a statistical analysis of different local government departmental collaborations in the same period can reveal the structural law of the coordination of the main body. In view of this, this research uses the $\mathrm{R}$ language to excavate the "13th Five-Year" Comprehensive Work Plan for Energy Conservation and Emission Reduction (hereinafter referred to as Plan) of 121 local governments, and analyzes the departmental collaborations in the Plan, reveals the main problems in energy conservation, and provides a reference for improving the energy efficiency of local governments.

\section{Data Processing and Research Method}

\subsection{Data Source and Processing}

\subsubsection{Data Source}

This study collects the Plan successively formulated by local governments since 2016 as the text data source, selects 2016 and 2018 GDP and total energy consumption data from local statistical yearbooks, and uses energy consumption per 10,000 yuan of GDP as energy efficiency. For reasons of data availability, a total of 121 local government data were collected.

\subsubsection{Data Processing}

The procedures for data cleaning are: (1) Clean up responsibilities. The responsibilities of local governments will be uniformly expressed. (2) Abbreviate and code responsibilities. For example, "promoting the transformation and upgrading of traditional industries" is abbreviated as "industrial upgrading" and the code is CYSJ. (3) Clean up, abbreviate, and code government departments. The abbreviation is used to display results, and the code is used for data mining. Departments and its abbreviation are listed as appendix. (4) Using departmentresponsibility as the mining data, the mining data of all local governments is combined into a data set. The data set contains 6897 mining data.

\subsection{Research Method}

\subsubsection{Association Rule Mining}

The research uses the $\mathrm{R}$ language, and uses the Apriori algorithm in the arules tool to mine the association rules of the data set, mine the cross-departmental relationship, and generate the departmental relationship table.

\subsubsection{Social Network Analysis}

Social networks could display collaborative relationships. The research uses the $\mathrm{R}$ language and igraph tools, this paper performs social network analysis on the departmental relationship table. In general, social network analysis is used to analyze the interrelationships between individuals in the society. The existence of relations is equal to 1 , and the absence of relations is equal to 0 , that is, the threshold is 1 . Since the policy text mining is a multi-value relationship table, it is necessary to convert the multi-value relationship table into a binary relationship table before performing social network analysis. As far as the departmental collaboration of multiple local governments is concerned, a kind of departmental collaboration even in the same place will also appear several times in government policy texts. The previous threshold setting was low. Departmental 
collaborations must be present in at least half of the local government policy texts before they can be considered to exist in a general sense. Otherwise, they can only be individual cases. This study uses the median 61 of 121 samples as the threshold. If the threshold is exceeded, the departmental collaborations are considered, in order to build a departmental collaborative core network. Exceeding the threshold means that the departmental collaboration exists in the general sense, and below the threshold is that the departmental collaboration does not exist in the general sense. Because the departmental collaboration that this research focuses on is the direct connection between nodes.

(1) Network density. The network density is the ratio of the actual relationship number. The greater the network density, the closer the relationship between nodes. Formula (1) is as follows.

$$
\text { Network Density }=\frac{L}{N(N-1) / 2}
$$

In the formula, $L$ is the number of connected edges in the network, and $N$ is the number of nodes in the network.

(2) Degree of centrality. The degree of centrality measures the importance of a node in the network. It is the number of nodes directly contacts other nodes in the network. The bigger the degree of centrality, the more important the position of the node in the network. Formula (2) is as follows.

$$
C_{D}\left(N_{i}\right)=\sum_{j=1}^{n} x_{i j}(i \neq j)
$$

In the formula, $C_{D}\left(N_{i}\right)$ represents the degree of centrality of node $i, x_{i j}$ is the direct connection between node $\mathrm{i}$ and $j, \sum x_{i j}$ is the number of direct connections between node $i$ and other $n-1$ nodes, and $n$ is the number of nodes in the network.

\subsubsection{Correlation Analysis}

Two kinds of collaboration structure rules can be explored through the correlation analysis of the collaboration frequency and energy efficiency.

\section{Analysis of Departmental Collaborations in Energy Conservation of Local Governments}

\subsection{Analysis of the Status Quo of Departmental Collaboration Network in Energy Saving}

Based on the departmental relationship table, this research conducted the following analysis on the departmental collaboration network in energy conservation:

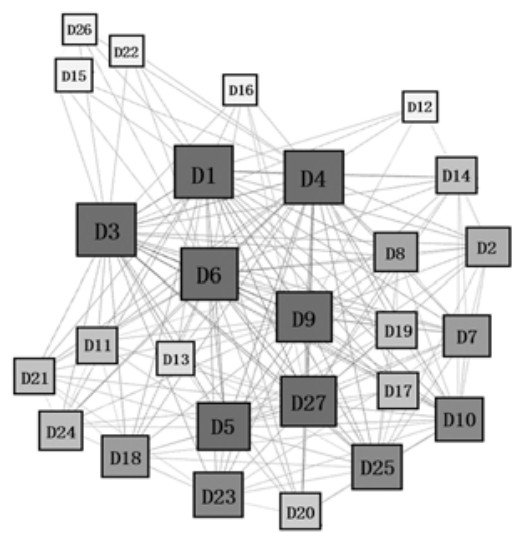

Figure 1. The core network of the departmental collaborations in energy conservation of local governments.

Figure 1 shows the status of the collaborative core network of local government departments where the cooccurrence frequency between departments exceeds the threshold. The core network scale includes 27 departments with a network density of 0.547 , which is a medium-to-high-density network, indicating that the closeness of the departmental collaboration is good. The general network scale includes 49 departments, and the network density is 0.529 . It is also a medium-to-highdensity network. The general network has 22 government departments more than the core network, but the closeness of departmental collaboration is only slightly lower. It shows that there are 27 departments that have commonality in energy conservation, and that 22 departments and other departments are only individual phenomena. The core network is consistent with the general network in the main government departments. They are Department of Industry and Information Technology(D4), Department of Development and Reform(D3), and Department of Ecology and Environment(D1). The point centralities are 26, 26, 26 and 47, 46, 45, respectively, indicating that these three government departments have collaborations with most other government departments no matter in the general network or in the core network. It also shows from one side that the close degree of collaborations between the general network and the core network department is basically the same, mainly due to the uncommon collaborations between 27 departments and 22 departments in the general network, while there is rarely any collaboration among 22 departments.

Table 1. Correlation analysis table of departmental collaborations and energy efficiency.

\begin{tabular}{|c|c|c|}
\hline $\begin{array}{c}\text { Departmental } \\
\text { Collaboration }\end{array}$ & $\begin{array}{c}\text { Correlation } \\
\text { Coefficient }\end{array}$ & P Value \\
\hline D4-D11 & -0.299 & 0.019 \\
\hline D4-D28 & -0.274 & 0.033 \\
\hline D1-D19 & 0.263 & 0.041 \\
\hline D1-D28 & -0.274 & 0.033 \\
\hline D7-D27 & -0.259 & 0.044 \\
\hline D7-D24 & -0.356 & 0.005 \\
\hline D7-D28 & -0.279 & 0.030 \\
\hline D6-D24 & -0.313 & 0.014 \\
\hline D6-D28 & -0.300 & 0.019 \\
\hline D23-D19 & 0.282 & 0.028 \\
\hline
\end{tabular}




\begin{tabular}{|c|c|c|}
\hline D23-D18 & -0.290 & 0.023 \\
\hline D23-D8 & -0.303 & 0.018 \\
\hline D23-D24 & -0.337 & 0.008 \\
\hline D5-D11 & -0.323 & 0.011 \\
\hline D5-D21 & -0.306 & 0.017 \\
\hline D5-D24 & -0.264 & 0.039 \\
\hline D5-D28 & -0.320 & 0.012 \\
\hline D25-D11 & -0.322 & 0.011 \\
\hline D25-D28 & -0.517 & 0.000 \\
\hline D19-D10 & 0.280 & 0.029 \\
\hline D19-D27 & 0.321 & 0.012 \\
\hline D19-D18 & 0.275 & 0.032 \\
\hline D19-D29 & 0.417 & 0.001 \\
\hline D19-D21 & 0.313 & 0.014 \\
\hline D19-D24 & 0.282 & 0.028 \\
\hline D19-D13 & 0.391 & 0.002 \\
\hline D9-D18 & -0.287 & 0.025 \\
\hline D9-D24 & -0.255 & 0.047 \\
\hline D10-D18 & -0.284 & 0.026 \\
\hline D10-D11 & -0.362 & 0.004 \\
\hline D10-D29 & 0.417 & 0.001 \\
\hline D10-D24 & -0.322 & 0.011 \\
\hline D27-D18 & -0.280 & 0.029 \\
\hline D27-D11 & -0.254 & 0.048 \\
\hline D27-D24 & -0.283 & 0.027 \\
\hline D2-D12 & 0.266 & 0.039 \\
\hline D18-D8 & -0.291 & 0.023 \\
\hline D18-D24 & -0.291 & 0.023 \\
\hline D11-D8 & -0.339 & 0.008 \\
\hline D11-D13 & -0.517 & 0.000 \\
\hline D8-D24 & -0.376 & 0.003 \\
\hline D8-D13 & -0.275 & 0.032 \\
\hline D26-D16 & -0.260 & 0.043 \\
\hline D29-D13 & 0.279 & 0.029 \\
\hline D21-D24 & -0.265 & 0.039 \\
\hline & & \\
\hline & & \\
\hline
\end{tabular}

\subsection{Analysis on the problems of departmental collaboration network in energy saving}

From the perspective of structural law, Table 1 shows that a total of 45 types of departmental collaborations have a significant correlation with energy efficiency. Among them, 12 types of departmental collaborations are significantly positively correlated with energy efficiency, indicating that as the frequency of collaborations between these 12 types of departments increases, energy efficiency improves. On the other hand, there are 33 types of departmental collaborations and energy efficiency are significantly negatively correlated, indicating that as the frequency of collaborations among these 33 types of departments increases, energy efficiency decreases. Among them, Department of Water Resources(D19) has the most positive correlations. This is because Department of Water Resources is involved in many areas of energy conservation. With the scarcity of water resources, the situation of using energy consumption in exchange for water source protection is very severe. Strengthening collaborations with Department of Water Resources can improve energy efficiency. Department of Culture and Tourism(D11) and Department of Education(D18) involve the most negative correlations. This is because culture, tourism and education are industries with low energy consumption, high output, and more employment. Strengthening collaborations with these two departments will inhibit the development of them under multiple controls. Government departments pay a higher cost of collaborations, which in turn leads to a reduction in energy efficiency.

Revealing the problem of departmental collaborations in local government energy conservation should not only be based on structural laws, but also based on the actual situation of departmental collaboration networks. According to the synergy theory, under the premise of keeping the environmental control variables unchanged, if the order parameter is formed in the self-organization process of energy conservation in government departments, then there will be a structural law for energy conservation in government departments. Due to the existence of the slaving principle, the collaborations will develop in the direction of improving energy efficiency [8]. This shows that there are objective laws in departmental collaborations of local governments, and the main problems in the existing departmental collaborations of local governments should be solved based on structural laws. Human intervention can save time and resources in the process of long-term competition, while the slaving principle should be used to solve the general problems in departmental collaborations. In Table 1, the collaborations between Department of Water Resources and Department of Assets Supervision and Administration(D23), Department of Education(D18), Commission Office of Public Sectors Reform of Local Government(D29), Local Publicity Department of the CPC(D21), Department of Radio and Television(D24), and Department of Human Resources and Social Security(D13), as well as the collaborations between Commission Office of Public Sectors Reform of Local Government(D29) and Department of Transport(D10), Department of Human Resources and Social Security(D13) are significantly positively related to energy efficiency. However, no connection is shown in figure 1, indicating that collaborations of these departments are not common phenomenon and there are serious shortcomings. Among them, Department of Water Resources has the most lack of collaborations, indicating that the main problem of departmental collaborations at this stage is that the local government ignores the important role of water conservation in energy conservation and does not pay attention to the collaborations of Department of Water Resources with other departments, which affects the improvement of energy efficiency. 


\section{Conclusion}

Under the influence of external environmental factors, local government departments will produce order parameters through self-organization, and structural laws have appeared in departmental collaborations. The existing departmental collaborations are governed by structural laws to improve energy efficiency. Some of the structural laws promote departmental collaborations, while others hinder departmental collaborations. Therefore, existing departmental collaborations should be adjusted on the basis of structural laws. Since the objective laws are mandatory, there is no need for human intervention to solve all departmental collaboration problems. We should focus on solving the main problems in departmental collaborations to save a lot of time and resources. The rest are made by the slaving principle.

This research reveals the structural law of departmental collaborations and the problems of departmental collaborations in local government energy conservation. Among the structural laws of departmental collaborations, Department of Water Resources involves the most positive correlations, and Department of Culture and Tourism and Department of Education involve the most negative correlations. The objective law shows that the collaboration with Department of Water Resources should be strengthened, and the collaborations with Department of Culture and Tourism and Department of Education should be reduced to improve energy efficiency. At this stage, the main problem of departmental collaboration is that Department of Water Resources is facing obvious lack of collaborations. Local governments have neglected the important role of water saving in energy conservation in energy conservation and should strengthen collaborations with Department of Water Resources.

\section{Appendix}

Abbreviation of local government departments.

\begin{tabular}{|c|c|}
\hline Local Government Departments & Abbreviation \\
\hline Department of Ecology and Environment & D1 \\
\hline Department of Natural Resources & D2 \\
\hline Department of Development and Reform & D3 \\
\hline $\begin{array}{c}\text { Department of Industry and Information } \\
\text { Technology }\end{array}$ & D4 \\
\hline Department of Market Regulation & D5 \\
\hline Department of Finance & D6 \\
\hline Department of Science and Technology & D7 \\
\hline Department of Commerce & D8 \\
\hline $\begin{array}{c}\text { Department of Housing and Urban-Rural } \\
\text { Development }\end{array}$ & D9 \\
\hline Department of Transport & D10 \\
\hline Department of Culture and Tourism & D11 \\
\hline $\begin{array}{c}\text { Department of Forestry and Grassland } \\
\text { Administration }\end{array}$ & D12 \\
\hline $\begin{array}{c}\text { Department of Human Resources and Social } \\
\text { Security }\end{array}$ & D13 \\
\hline
\end{tabular}

\begin{tabular}{|c|c|}
\hline $\begin{array}{c}\text { Department of Agriculture and Rural } \\
\text { Affairs }\end{array}$ & D14 \\
\hline People's Bank of China & D15 \\
\hline Department of Taxation & D16 \\
\hline Department of Energy & D17 \\
\hline Department of Education & D18 \\
\hline Department of Water Resources & D19 \\
\hline Local Organization Department of the CPC & D20 \\
\hline Local Publicity Department of the CPC & D21 \\
\hline $\begin{array}{c}\text { Department of Financial Supervision and } \\
\text { Administration }\end{array}$ & D22 \\
\hline $\begin{array}{c}\text { Department of Assets Supervision and } \\
\text { Administration }\end{array}$ & D23 \\
\hline Department of Radio and Television & D24 \\
\hline Department of Statistics & D25 \\
\hline $\begin{array}{c}\text { Local Banking and Insurance Regulatory } \\
\text { Commission }\end{array}$ & D26 \\
\hline $\begin{array}{c}\text { Department of Local Government Offices } \\
\text { Administration }\end{array}$ & D27 \\
\hline Department of Emergency Management & D28 \\
\hline $\begin{array}{c}\text { Commission Office of Public Sectors } \\
\text { Reform of Local Government }\end{array}$ & D29 \\
\hline \begin{tabular}{c} 
Derm \\
\hline
\end{tabular} & \\
\hline
\end{tabular}

\section{References}

1. S. Zadek, Corp. Gov. Intl. J. Biz. Soc 8, 374-388 (2008)

2. P. Hicks, D. Ritter, K. Seltzer, G. Stoker, Towards Holistic Governance: The New Reform Agenda (Palgrave Assoc, Lon, 2002)

3. E. Meijers, D. Stead, Ber. Greening of PoliciesInterlinkages and Policy Integration (Ber, 2004)

4. A. Matei, T.C. Dogaru, Procedia Soc and Behav Sci 81, 65-71(2013)

5. M. J. Hoel, Pub. Econ 66, 199-224(1997)

6. X.J. Cao, J.W. Li, SMS, 84-93 (2019)

7. J.X. Li, X.G. Zhang, Y. Hu, Z.L. Chen, J.H. Yin, H.D. Feng, SE-T\&P, 37, 1499-1511(2017)

8. Y.J. Bao, Thu. Biz. Rev, 6-19(2019) 